\section{Hilft Hirndoping?}

Das Thema Hirndoping oder "brain enhancement " taucht zunehmend in der Laienpresse auf, in der Fachliteratur wird jedoch noch wenig darüber berichtet. Der Autor, Prof. Dr. Laus Lieb, Psychiater und Psychotherapeut, beschäftigt sich seit Jahren mit dem Thema Hirndoping und Psychopharmaka. In seinem Buch diskutiert er Motive für Hirndoping und seine Grenzen. Er spannt den Bogen aber noch weiter bis in die Zukunft hinein: Zukunftsmarkt für Hirndoping, seine ethischen Aspekte - und auch Alternativen.

Der Autor lässt von Beginn an keine Zweifel an seiner Meinung: Er hält eine Legalisierung des Hirndopings, wie sie von einem deutschen Expertengremium, dem deutschen Ethikrat, in einem Eckpunktepapier 2009 empfohlen wurde, für falsch. Hier bezieht er sich jedoch vor allem auf durch Psychopharmaka durchgeführtes „brain enhancement". Laut seiner eigenen Definition von Hirndoping wird darunter der Versuch gesunder Menschen, die Leistungsfähigkeit des Gehirns durch die Einnahme von verschreibungspflichtigen Medikamenten zu verbessern, verstanden. Eine Steigerung der geistigen Leistungsfähigkeit durch Koffein oder pflanzliche Produkte, die frei verkäuflich sind, stellt aus seiner Sicht kein Hirndoping dar. Warum nur verschreibungspflichtige Mittel und nicht frei erhältliche Mittel zu einem Doping des Gehirns führen sollen, lässt er offen. Hier wäre eine allgemein akzeptierte Definition erforderlich.

Detailliert beschreibt der Autor Studien über Veränderung der geistigen Leistungsfähigkeit gesunder Menschen unter Psychopharmaka, insbesondere Stimulanzien, die therapeutisch bei ADHS eingesetzt werden, sowie Antidepressiva. Er zitiert zunächst korrekt, dass keine kognitive Verbesserung der Leistungsfähigkeit unter den bisher erhältlichen Psychopharmaka auftritt. Lediglich eine erhöhte „Wachheit" wird unter den Stimulanzien und auch Modafinil, das therapeutisch für Narkolepsie eingesetzt wird, beobachtet. Er schlussfolgert aber, dass Wachheit mit Konzentration und Aufmerksamkeit zusammenhänge und daraus eine Leistungssteigerung resultiere. Es gibt sie also nicht, noch nicht: die Superpille.

Zweifelsfrei ist aber, dass bereits gesunde Menschen Psychopharmaka zum Ziele der Leistungssteigerung einnehmen: In einer Umfrage aus dem Jahr 2008 in der Zeitschrift "Nature" geben $20 \%$ der Befragten an, bereits eines oder mehrere Medika- mente zum kognitiven Enhancement eingenommen zu haben, davon $12 \%$ Stimulanzien [Greely $\mathrm{H}$ et al. Nature 2008; 456 : 702-5]. Bei einer Befragung von 600 Schülern aus einer Mainzer Studie, geben 1-2\% der 18-21-Jährigen an, bereits mindestens einmal in ihrem Leben Hirndoping mit verschreibungspflichtigen Substanzen versucht zu haben. Über $50 \%$ geben auf Befragung an, dass sie, sollte es eine wirksame und verfügbare Substanz zur Leistungssteigerung geben, diese auch einnehmen würden, falls nicht mit gefährlichen Nebenwirkungen zu rechnen sei.

Auch wenn es die Wunderpille aktuell nicht gibt, die aus uns allen kleine Einsteine macht, so ist es möglicherweise nur eine Frage der Zeit. Wie aber soll dann damit umgegangen werden? Wie sind die ethischen Grundlagen, wie die rechtlichen? Was bedeutet es für den Arzt, vor allem bei fließenden Grenzen zwischen Therapie und Enhancement? Am Muskeldoping im Sport sehen wir, wie wichtig es ist, zeitig Diskussionen zu führen und gegebenenfalls Kontrollinstanzen zu errichten, um zu verhindern, dass Pharmafirmen in Zukunft die guten Noten schreiben und erste Plätze bei den Prüfungen ablegen.

Das Buch vermittelt eine gute Übersicht über zurzeit vorliegende wissenschaftliche Daten, es regt zum Nachdenken an und führt hoffentlich dazu, dass sich diesem komplexen Thema genähert wird - frei von Ideologien.

Dr. Kirsten Stollhoff

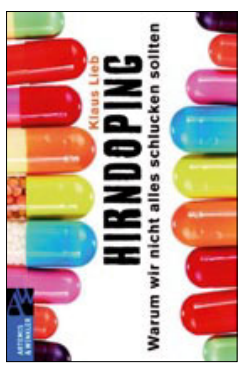

Klaus Lieb

Hirndoping

- warum wir nicht alles schlucken sollen

Verlag Artemis \& Winkler, Mannheim 2010

$16,90 €$

ISBN 3-538-07301-5

Erratum

In der letzten Ausgabe hatten sich im Beitrag "Zusatzleistungen in der Pädiatrie - Clever für Kids" [Zieriacks P. pädiatrie hautnah 2011; 23 (5): 420-1] Fehler in der Tabelle eingeschlichen. Eine korrigierte und aktualisierte Fassung der Tabelle, aus der hervorgeht, welche Krankenkassen am Zusatzvertrag "Clever für Kids" teilnehmen, finden sie online unter www.springermedizin.de/cleverfuer-kids/2255066.html. 